\title{
Narrowband optical interactions in a plasmonic nanoparticle chain coupled to a metallic film
}

\author{
Daniele Brunazzo, ${ }^{1,2, *}$ Emiliano Descrovi, ${ }^{2}$ and Olivier J. F. Martin ${ }^{1}$ \\ ${ }^{1}$ Nanophotonics and Metrology Laboratory, Swiss Federal Institute of Technology Lausanne (EPFL), EPFL-STI-NAM, \\ ELG Station 11, CH-1015 Lausanne, Switzerland \\ ${ }^{2}$ Microsystems and Material Laboratory- $-\chi$ LabPolitecnico di Torino, corso Duca degli Abruzzi 24, 10129 Torino, Italy \\ *Corresponding author: daniele.brunazzo@epfl.ch
}

Received January 30, 2009; accepted March 18, 2009; posted March 30, 2009 (Doc. ID 106992); published April 24, 2009

\begin{abstract}
We study the coupling of localized surface plasmon (LSP) and surface-plasmon polariton (SPP) modes in a system composed of a metallic nanoparticle chain separated from a thin metallic film by a dielectric spacer. The thickness of such a spacer influences the level of interaction between LSP and SPP modes and controls the electromagnetic enhancement in this system. An enhancement with narrow resonances can be observed for appropriate parameters. The high-resonance quality factor and tunability of this system make it a very promising candidate for biosensing and surface-enhanced spectroscopy applications. (C) 2009 Optical Society of America

OCIS codes: $240.6680,350.4238,350.4990$.
\end{abstract}

During the past few decades the optical response of ensembles of noble metal nanoparticles has been a subject of interest in different fields, from optoelectronics to biophotonics [1,2]. Single metal nanoparticles exhibit characteristic localized surface plasmon (LSP) modes that depend on different parameters such as the nanoparticle size, composition, shape, and surrounding medium [3]. By placing two or more plasmonic nanoparticles close to each other it is possible to observe the interaction between the modes of each individual nanostructure leading to the formation of new hybrid modes [4].

When several nanoparticles are arranged in a onedimensional (1-D) linear chain, the interaction of the electromagnetic field associated with each single particle can produce several plasmonic chain modes with interesting properties [5]. The local enhancement and the squeezing of the electromagnetic field at the proximity of these metallic chains have been experimentally demonstrated $[6,7]$ and used to realize extremely thin subwavelength optical waveguides $[8,9]$. The behavior of a 1-D metallic nanoparticle chain embedded in homogeneous media has been studied theoretically by Schatz et al. [10] and experimentally verified [11]. This system presents a shift of the resonance wavelength that depends on the chain's parameters, such as the spacing between the nanoparticles, their orientation with respect to the wave vector, and the polarization of the electromagnetic field. In addition, coherent interaction arising from multiple scattering by regularly spaced nanoparticles can result in narrow plasmon resonances and sharp peaks $[10,12]$.

Surface plasmon-polariton (SPP) modes are surface waves confined at a metal-dielectric interface originating from the resonant excitation of the free electrons in the metal [13]. The coupling between LSP and SPP modes displays interesting characteristics, and a dramatic enhancement of the electromagnetic field has been predicted for the case of one single metallic nanoparticle interacting with a metallic substrate [14]. Also, the presence of a metallic sub- strate at the proximity of a metallic nanoparticle chain can significantly increase the propagation length of the plasmonic modes guided by the chain [15].

In this Letter, we study numerically the behavior of a metallic nanoparticle chain coupled to a thin metallic film deposited on a silica substrate. Specifically, we show how the interaction between SPP and LSP supported by this system can be tuned by changing the thickness of a silica spacer introduced between the two plasmonic structures. The dispersion curves of both SPP and LSP modes derived from the absorption coefficient of the nanoparticle chain are used to explain the coupling mechanism. Absorption spectra and field distributions evidence an enhancement of the electromagnetic field. Calculations are performed with the Green's tensor method described in $[16,17]$.

The system under study consists of a metallic nanoparticle chain separated from a thin gold film by a silica spacer with thickness $t$ (Fig. 1). A 1-D chain with 100 spherical gold particles (radius, $20 \mathrm{~nm}$; period, $260 \mathrm{~nm}$ ) is considered. The gold film is $40 \mathrm{~nm}$ thick, and the permittivity as a function of the wavelength for both the particles and the film is taken from [18]. The permittivity of silica has been set to $\varepsilon=2.25$. A $p$-polarized plane wave illuminates the whole system through the silica substrate, with the

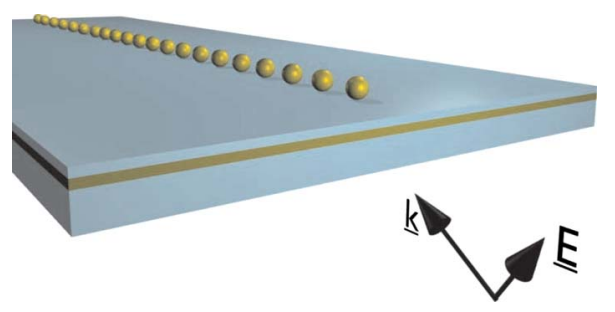

Fig. 1. (Color online) System under study: metallic nanoparticle chain deposited on a stratified $\mathrm{SiO}_{2}-\mathrm{Au}-\mathrm{SiO}_{2}$ substrate. The interaction between delocalized SPP modes propagating on the film with LSP chain modes leads to an enhancement of the electromagnetic field. 
projection of the wave vector on the substrate parallel to the chain axis; see Fig. 1.

The optical response of a simple gold nanoparticle chain lying on a glass substrate is shown in Fig. 2(a). Owing to the localized character of LSP on finitesized objects, this optical resonance has a nondispersive behavior in the $\theta-\lambda$ plane. Here, the resonance exhibits one fundamental peak around $\lambda=513 \mathrm{~nm}$.

The typical SPP dispersion curve appears when the stratified layer consisting of a silica spacer, and a thin gold film is placed under the nanoparticle chain. Such a dispersion curve can be shifted toward longer wavelengths by varying the thickness of the silica spacer, similarly to the wavelength shifts observed in other surface wave phenomena [19]. When the resonances of both SPP and LSP modes occur at the same wavelength, the strong interaction between these two modes gives rise to an enhancement of the electromagnetic field. In Fig. 2 it is possible to observe the influence of the silica spacer thickness on the crossing point of the two modes. In the case of a very thin spacer $(t=5 \mathrm{~nm})$ this interaction is clearly visible on the absorption map in Fig. 2(b): The coupling between both modes taking place in the broad region around $\theta=45^{\circ}$ is a direct consequence of the excitation of LSP modes on the chain by the SPP mode propagating at the upper gold-silica interface.

The dispersion curve of the SPP shifts toward lower wavelengths when the thickness of the silica spacer increases; compare Figs. 2(b) and 2(d). Hence, the coupling strength between SPP and LSP modes changes with the thickness $t$. The maximal coupling is observed for $t=200 \mathrm{~nm}$ and $\theta=42^{\circ}$, as shown in Fig. 2(c). This maximum coupling produces an enhancement and narrowing of the resonance peak. In contrast, Fig. 2(d) shows the behavior of a weakly coupled system, where the interaction between the
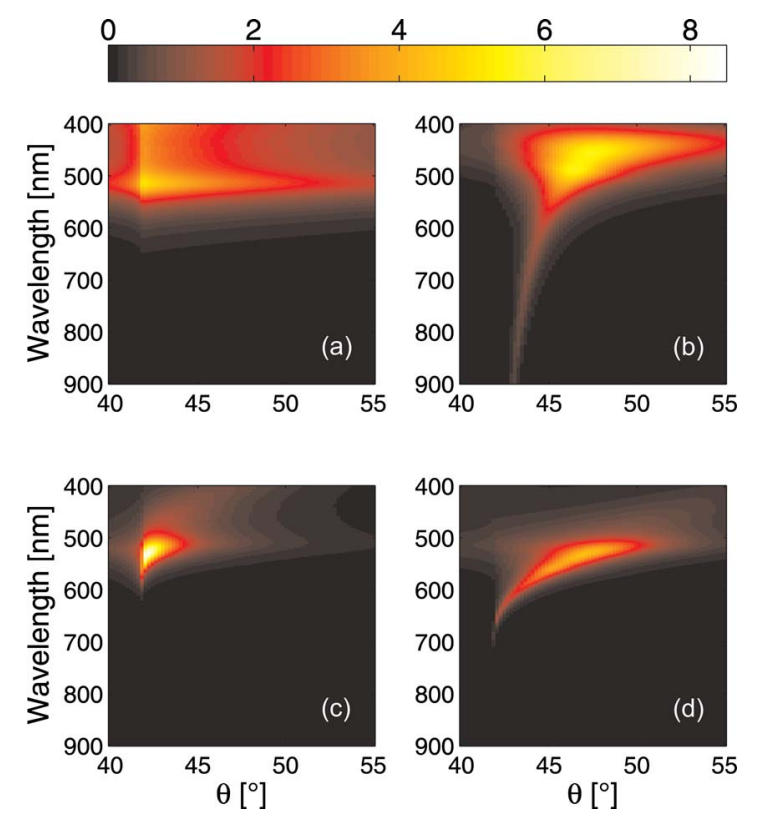

Fig. 2. (Color online) Absorption maps as a function of the illuminating wavelength $\lambda$ and the angle of incidence $\theta$ for different geometries: (a) no gold film, (b) $5 \mathrm{~nm} \mathrm{SiO}_{2}$ spacer, (c) $200 \mathrm{~nm} \mathrm{SiO}$ spacer, and (d) $260 \mathrm{~nm} \mathrm{SiO}_{2}$ spacer. chain and the substrate is reduced: The dispersion curve of the SPP is clearly visible and nearly unchanged by the particle chain, the amplitude of its resonance peak not differing significantly from the case of a chain lying on a simple glass substrate.

Figure 3 shows a comparison between the resonance of a gold nanoparticle chain placed in different environments: a homogeneous dielectric medium (air, $\varepsilon_{\text {air }}=1$ ), a semi-infinite silica substrate, and finally a stratified $\mathrm{SiO}_{2}-\mathrm{Au}-\mathrm{SiO}_{2}$ substrate $\left(200 \mathrm{~nm} \quad \mathrm{SiO}_{2}\right.$ spacer). The latter two cases correspond to the situations shown in Figs. 2(a) and 2(c), respectively. The interaction between SPP and LSP modes induced by the presence of a thin gold film produces a spectral shift $(\sim 20 \mathrm{~nm})$, together with a narrowing and an enhancement of the resonance peak.

Furthermore, it is possible to estimate such an enhancement by looking at the intensity of the electromagnetic field for these different geometries. In Fig. 4 the cases of a plasmonic chain lying on top of a semi-infinite silica substrate and on a stratified $\mathrm{SiO}_{2}-\mathrm{Au}-\mathrm{SiO}_{2}$ substrate are considered: For clarity's sake, only the field profiles calculated above the two central particles of the chain are shown. An enhancement factor of $\simeq 2.5$ is observed: This phenomenon is due to the direct excitation of LSP chain modes by SPP modes, resulting in a considerable increase of the electromagnetic field in the proximity of the metallic nanoparticle chain. Field maxima are located close to the nanoparticles' surface, as outlined in [6].

Sharp resonance peaks at different positions can thus be observed by varying the geometrical parameters of such a system. Such control over the LSPSPP coupling phenomenon can be exploited for specific applications, where the spectral response of the system can be tuned to engineer, e.g., hot sites for surface-enhanced spectroscopy. All these features render this system extremely promising for the development of reliable and ultrasensitive biochemical sensors [20].

In conclusion, in this Letter we have studied the coupling between LSP and SPP modes in a system

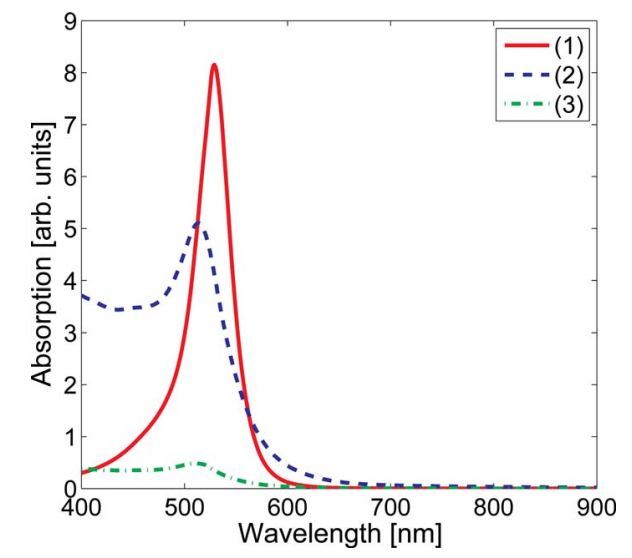

Fig. 3. (Color online) Absorption spectra of a gold nanoparticle chain: (1) on a $\mathrm{SiO}_{2}-\mathrm{Au}-\mathrm{SiO}_{2}$ substrate with a $200 \mathrm{~nm} \mathrm{SiO}_{2}$ spacer, (2) on a semi-infinite $\mathrm{SiO}_{2}$ substrate without $\mathrm{Au}$ film, (3) embedded in a homogeneous medium (air, $\varepsilon=1$ ). 


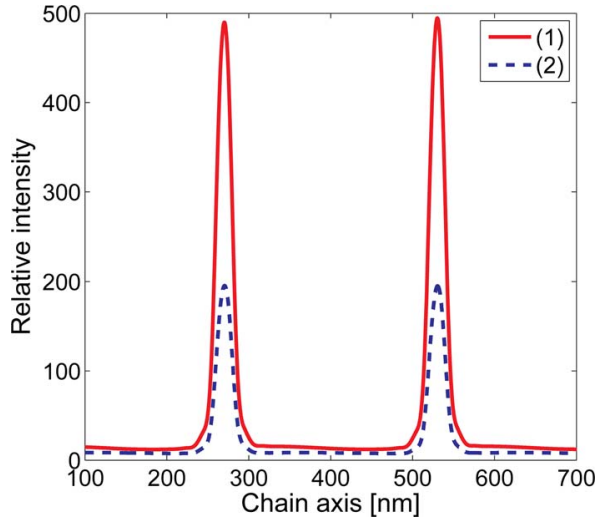

Fig. 4. (Color online) Intensity distributions $40 \mathrm{~nm}$ above two particles of a gold plasmonic chain: (1) on a $\mathrm{SiO}_{2}-\mathrm{Au}-\mathrm{SiO}_{2}$ substrate with a $200 \mathrm{~nm} \mathrm{SiO}_{2}$ spacer, (2) on a semi-infinite $\mathrm{SiO}_{2}$ substrate without $\mathrm{Au}$ film.

composed of a gold particle chain coupled to a thin gold film. We have demonstrated that the field enhancement as produced by LSP on periodic arrangements of metallic nanoparticles can be increased by exploiting a concurrent excitation of SPP. Sharp resonance peaks and electromagnetic enhancement have been observed, leading to a highly tunable system for biosensing and surface-enhanced spectroscopy. We believe that such an enhancement might be further improved by using 1-D photonic crystals as a substrate in place of the gold film. We argue that the high-intensity surface modes [21] sustained by 1-D photonic crystals can represent an additional way for boosting the nanoparticle chain resonance factor.

Funding from the Swiss National Competence Center in Research Nanoscale Science and from the Centre of Excellence funded by the Italian Ministry of University and Research (MIUR) Laboratorio di Tecnologie Elettrobiochimiche Miniaturizzate per l'Analisi e la Ricerca (grants FIRB 2003-2004) are gratefully acknowledged.

\section{References}

1. S. A. Maier, P. G. Kik, H. A. Atwater, S. Meltzer, E. Harel, B. E. Koel, and A. A. G. Requicha, Nature Mater. 2, 229 (2003).
2. J. N. Anker, W. P. Hall, O. Lyandres, N. C. Shah, J. Zhao, and R. P. Van Duyne, Nature Mater. 7, 442 (2008).

3. J. P. Kottmann, O. J. F. Martin, D. R. Smith, and S. Schultz, Phys. Rev. B 64, 235402 (2001).

4. E. Prodan, C. Radloff, N. J. Halas, and P. Nordlander, Science 302, 419 (2003).

5. K. B. Crozier, E. Togan, E. Simsek, and T. Yang, Opt. Express 15, 17482 (2007).

6. J. R. Krenn, A. Dereux, J. C. Weeber, E. Bourillot, Y. Lacroute, J. P. Goudonnet, G. Schider, W. Gotschy, A. Leitner, F. R. Aussenegg, and C. Girard, Phys. Rev. Lett. 82, 2590 (1999).

7. P. Ghenuche, I. G. Cormack, G. Badenes, P. LozaAlvarez, and R. Quidant, Appl. Phys. Lett. 90, 041109 (2007).

8. C. Girard, E. Dujardin, M. Li, and S. Mann, Phys. Rev. Lett. 97, 100801 (2006).

9. F. Bonell, A. Sanchot, E. Dujardin, R. Pechou, C. Girard, M. Li, and S. Mann, J. Chem. Phys. 130, 034702 (2009).

10. S. Zou and G. C. Schatz, J. Chem. Phys. 121, 12606 (2004).

11. E. M. Hicks, S. Zou, G. C. Schatz, K. G. Spears, R. P. VanDuyne, L. Gunnarson, T. Rindzevicius, B. Kasemo, and M. Kall, Nano Lett. 5, 1065 (2005).

12. S. Zou and G. C. Schatz, Nanotechnology 17, 2813 (2006).

13. W. L. Barnes, A. Dereux, and T. W. Ebbesen, Nature 424, 824 (2003).

14. G. Lévêque and O. J. F. Martin, Opt. Express 14, 9971 (2006).

15. G. Lévêque and R. Quidant, Opt. Express 16, 22029 (2008).

16. O. J. F. Martin and N. B. Piller, Phys. Rev. E 58, 3909 (1998).

17. M. Paulus and O. J. F. Martin, J. Opt. Soc. Am. A 18, 854 (2001).

18. P. B. Johnson and R. W. Christy, Phys. Rev. B 6, 4370 (1972).

19. E. Descrovi, F. Giorgis, L. Dominici, and F. Michelotti, Opt. Lett. 33, 243 (2008).

20. C. R. Yonzon, D. A. Stuart, X. Zhang, A. D. McFarland, C. L. Haynes, and R. P. Van Duyne, Talanta 67, 438 (2005).

21. E. Descrovi, T. Sfez, L. Dominici, W. Nakagawa, F. Michelotti, F. Giorgis, and H.-P. Herzig, Opt. Express 16, 5453 (2008). 